\title{
CULTURALLY-SENSITIVE PSYCHIATRIC NURSING CARE: AN IMPORTANT PRELIMINARY INTERVENTION IN COMMUNITY-BASED ACUTE CARE SETTINGS
}

\author{
Randy Ian F. Gallego \\ College of Health Sciences - Mindanao State University, Main Campus \\ Marawi City, Philippines, 9700. \\ Correspondence Addres: Randy Ian F. Gallego \\ Email: randyian_17@yahoo.ph
}

\begin{abstract}
ABSTRACK
Psychiatric nursing care is an indispensable aspect of the total health care services provided by nurses in both the acute and chronic care settings. This study investigated the extent of preliminary clinical nursing care provided by registered nurses in dealing with clients who have unique psycho-behavioral needs. It further looked into the relationship between the nurses' socio-economic profile including their ethnic affiliation and the acute care services offered to patients admitted in the regular hospital, non-psychiatric healthcare settings. It used a descriptive-correlational design through a validated and reliability-tested instrument responded by 113 staff nurses from six hospitals around Lanao del Sur. It involved the statistical analysis of data using frequency, weighted mean, and Pearson correlation. The findings show that the nurse-respondents of the selected hospitals in Lanao del Sur generally considers the extent of implementation of the six nursing care areas (counseling, milieu therapy, self-care activities, psychobiologic interventions \& health-teachings, case management, health promotion and maintenance) as frequently done or as generally emphasized in about $75 \%$ of the time in their respective clinical areas. When tested at 0.05 level of significance, the $p$-value obtained was less than the critical value $(\mathrm{p}=0.00$ 0.006) revealing that the ethnic affiliation of nurses was significantly correlated to the health promotion and maintenance care that they provide to clients with psycho-behavioral pathologies. Therefore, nurses serving in acute clinical settings need to possess a significant amount of competency in psychiatric care to be able to render preliminary interventions despite non-specialist background. Nurses also have to be cognizant of the cultural history and background of the clients being served even at the preliminary phase of the nurse-patient interaction and accommodate them with nursing interventions based on transcultural competence.
\end{abstract}

Keywords: Culturally-sensitive psychiatric care, psycho-behavioral pathologies, preliminary psychiatric interventions, psychiatric care in acute settings

\section{INTRODUCTION}

Psychological illness and other related disturbances can significantly interfere with the daily activities of people affected by it. On average, it is estimated that more than half of the general population in developed countries will be afflicted by some form of mental health issue in their lifetime. Hence, there is a growing recognition that mental illness is indeed a major public health concern which can have significant societal impacts. Psychosocial and mental aberrations are associated with severe distress and functional disability. The consequences are not only known to affect the infirmed individual but also their families and their capacity to live and perform normal human activities (Patel et al., 2016).

Apparently, the need to look at mental illness as a pressing concern in today's healthcare sector should arouse not just the medical populace but also the national government to improve the standards in the preventive modalities and management of mental illness and its related disorders. Basing on a statistics gathered by the WHO-AIMS Report on Mental Health System in the Philippines in 2007 (Hamid et al., 2008), it was approximated that in 2005, there were about 22,745,162 cases of mental illness in the Philippines; however, there were only forty-six (46) outpatient institutions treating 124.3 users per 100,000 populations. The rate of users per 100,000 
general populations for day treatment facilities and community based psychiatric in-patient units are 4.42 and 9.98, respectively. There are fifteen (15) community-based residential (custodial home-care) facilities that treat 1.09 patients per 100,000 general populations. Mental hospitals manage about 8.97 patients per 100,000 general populations and the occupancy rate is $92 \%$. It was also noted that within the past five years, there has been no actual increment in the number of hospital beds to accommodate the everincreasing quantity of in-patient confinement.

What seems to be even more challenging in this setting is that there are not enough health workers who are specially trained to address the individual needs of these types of clients. According to the same study, in an effort to interfuse available mental health care delivery in community-based systems, the Philippine National Mental Health Program in the 1990 's promoted the exposure of public nurses and doctors by engaging them in training that would sharpen their competencies in the identification and management of psychiatric symptoms and their co-morbid mental disorders. Even so, it turned out that those who have undergone the community-based training have already vacated their work posts and those who are presently occupying the functions are not sufficiently prepared in terms of training and background to respond to the needs of the clienteles. There are also not enough physical resources available address psycho-social health needs.

In the Lanao del Sur province, for instance, no established mental healthcare agency has been instituted to cater to the needs of clients with mental health issues. To that effect, in terms of the country-wide profile, ARMM holds no regional in-patient mental facility. This fact also holds true to most of the hospital venues in Mindanao. It was even cited in a study that even at the turn of the new millennium, "the appointed Health Secretary himself, in one of his visits to inspect the various regional health facilities in the country, have witnessed for himself how miserable the conditions of patients are in public psychiatric agencies where patients were left to go around naked inside the premises while some were unattended and immobilized along the corridors" (Conde, 2004).

The dismal mental health condition in the locality is a phenomenon that is not exclusive in the country. Most developing nations continue to struggle to provide better health outcomes on the aspect of psycho-social care. According to a World Health Organization report in 2007, mental health and mental health nursing are still critical issues in most countries worldwide. Particularly, nursing practitioners in developing countries are the most usual frontliners in the care of patients with psycho-behavioral manifestations. They generally have different levels of training in their internship in mental health practice. While others are highly qualified in terms of their background and expertise in psychiatric nursing assessment, diagnosis, and interventions, there are also other professional nurses who lack exposure and skills in the area. In most instances, these nurses without adequate preparation are the only ones available in the healthcare settings to attend to the needs of patients whose clinical presentations are associated with psychiatric disorders. They often deliver fragmented interventions in isolated settings without support from other more experienced colleagues.

In any care setting where acute management of psychological impairment is evident in the client's symptomatologies, the role of the nurse traverses both the addressing of the actual and potential needs of the client. According to the Statement on Psychiatric-Mental Health Clinical Nursing Practice released by the American Nurses Association in 2014 (Kane, 2015), the phenomena of concern for nurses includes: the maintenance of optimal health and wellbeing and the prevention of psychobiologic illness, assessing and assisting the self-care 
limitations of clients, symptom management associated with psychopharmacologic intervention and treatment regimen, and providing support for deficits in functioning associated with emotional stress and anxiety.

On top of the provision of quality clinical care, certain factors are also to be considered in the discharge of nursing functions in acute settings. This involves making certain that appropriate cultural interventions are also afforded to clients as this is a crucial part of their holistic care. Competency in transcultural nursing is not only applicable in regular medical-surgical units but more so in the care of clients with psycho-behavioral pathologies.

Given this background, this study investigated the extent of preliminary clinical nursing care provided by registered nurses in dealing with clients with unique psychological needs. It is interesting to find out how local nurses are rendering their psychiatric nursing care in view of the aforementioned deficiencies in locale's healthcare system. It also examined the relationship between the two variables: extent of care provided and the sociodemographic profile of the nurserespondents, especially their ethnic background.

\section{METHODS}

The data utilized in this investigation was generated from a crosssectional, researcher-administered survey of 113 respondents from the randomly selected hospitals in Lanao del Sur. A descriptive correlation research design was selected to fulfill the objectives of the inquiry. The study was conducted in six (6) private and government hospitals which were geographically distributed around the province of Lanao del Sur. These six hospitals were chosen on the basis of their being considered as an operational, private or government-operated facilities equipped to attend to the preliminary health care needs of the general population of Lanao del Sur with a minimum primary healthcare facility status and more than 20-bed capacity patient coverage. Likewise, they were checked for feasibility in terms of organizational structure and accessibility to clients. And hence, they qualified the eligibility for study inclusion.

Registered Nurses were primed as main respondents in the study because they possess the legal mandate to be health providers and advocates of clients with diverse clinical needs, including psychiatric care. Hence, they are the ones expected to manage the patients' various health affairs including but not limited to the provision of preliminary mental health care thus enabling them to meet the inclusion criteria. To be eligible as respondents, these nurses should be: 1.) Current staff members of the selected hospital regardless of their employment status; and 2.) have taken care of client(s) with a reported or diagnosed case of psycho-behavioral pathology. Criteria 2 was determined using a presurvey of the profile of all nurses employed in the randomly selected hospitals. An item in the pre-survey tool specifically asked for the nurse's history and prior experience in handling clients with known psychobehavioral symptoms.

The research instrument was a survey-questionnaire composed of five (5) sections with a total of 123 items. A panel of experts composed of the panel members, validators, and the statistician were requested to review the content validity of the research instrument. The experts were asked to check each item in the questionnaire to test if they were specific and aligned to the study problems, and whether the items would sufficiently measure all the dimensions of the construct. Thus, comments and suggestions of the experts were acknowledged.

After all the validated data has been gathered, the results were then tested for Content Validity Ratio. The result of the tabulation of CVR was based on its standard corollary in which the score of 0.7 - 0.9 was Retained, $0.6-0.5$ Revised and 
$0.4-0.1$ was considered Rejected. Consequently, the researchers conducted a pilot study with 15 Staff Nurses from the study locale to subject the instrument for Cronbachs's Alpha Reliability Coefficient. For scientific study purposes, a helpful general principle is that reliability should be at least 0.70 or even higher" (Fraenkel et al, 2012).

Hence, in the test instruments of the study, the reliability of the survey instrument is 0.987 or $98.7 \%$. Data were then statistically treated using descriptive statistics with frequencies and percentages for qualitative variables and means and standard deviations for quantitative variables. Pearson correlation was utilized to determine the presence of relationships among the quantitative variables. Statistical significance was considered at p-value $\leq$

Table 1. Frequency Distribution of the Studied Sample According to their Personal Profile
.05. The informants' responses were tabulated separately with the use of the Statistical Package for Social Science (SPSS) version 20.

\section{RESULTS}

This part presents the collected data organized in tabular illustration. Similarly, tables and discussions are presented according to the following flow: frequency and percentage distribution of the sociodemographic profile of the respondents, descriptive statistics of the extent of preliminary clinical care provided by the nurses to clients with psycho-behavioral pathologies, and the correlation between the socio-demographic profile and extent of clinical care given.

\section{Characteristics (No. $=113$ )}

\begin{tabular}{|c|c|c|c|}
\hline Variables & & No. & $\%$ \\
\hline \multirow[t]{4}{*}{ Age Group } & 22 and below & 13 & 11.5 \\
\hline & $23-28$ & 70 & 61.9 \\
\hline & $29-34$ & 23 & 20.3 \\
\hline & 35 and up & 7 & 6.2 \\
\hline \multirow[t]{2}{*}{ Gender } & Male & 25 & 22.1 \\
\hline & Female & 88 & 77.9 \\
\hline \multirow[t]{5}{*}{ Civil Status } & Single & 81 & 71.7 \\
\hline & Married & 27 & 23.9 \\
\hline & Widowed & 3 & 2.7 \\
\hline & Divorced & 1 & 0.9 \\
\hline & Others & 1 & 0.9 \\
\hline \multirow[t]{4}{*}{ Ethnic Affiliation } & Meranao & 74 & 65.5 \\
\hline & Maguindanaon & 5 & 4.4 \\
\hline & Cebuano & 19 & 16.8 \\
\hline & Others & 15 & 13.3 \\
\hline \multirow[t]{4}{*}{ Religion } & Islam & 82 & 72.6 \\
\hline & Christian & 29 & 25.7 \\
\hline & Unaffiliated & 1 & 0.9 \\
\hline & Others & 1 & 0.9 \\
\hline \multirow[t]{4}{*}{ Monthly Income } & 20,000 Php Or More & 19 & 16.81 \\
\hline & 15,001 Php - 20,000 Php & 12 & 10.62 \\
\hline & 10,001 Php - 15,000 Php & 16 & 14.16 \\
\hline & 0,001 Php-10,000 Php & 66 & 58.41 \\
\hline \multirow[t]{5}{*}{ Length of Service } & 1 Year And Below & 47 & 41.6 \\
\hline & 1 Year \& 1 Month -2 Years & 19 & 16.8 \\
\hline & 2 Years \& 1 Month -3 Years & 16 & 14.2 \\
\hline & 3 Years \& 1 Month -5 Years & 15 & 13.3 \\
\hline & 5 Years And Above & 16 & 14.2 \\
\hline
\end{tabular}


The table shows that the general distribution of the nurse-respondents falls under the female, single, and young-adult age group. Most of them belong to the Meranao ethnic group, who are predominantly practitioners of Islam. More than half of the respondents have a monthly income of not more than 10,000 Php which is below the approved salary rate of Filipino nurses as stipulated in the Philippine Nursing Act of 2001. Many of them are novice nursing professionals with work experience of not more than one (1) year.

Table 2. Summary on the Extent of Preliminary Clinical Care provided to Clients with Psychobehavioral Symptoms

\begin{tabular}{lccc}
\hline & Preliminary Clinical Care on the Context of: & MEAN & $\begin{array}{c}\text { DESCRIPTIVE } \\
\text { RATING }\end{array}$ \\
\hline $\mathbf{1}$ & Counseling & 3.676 & Frequently \\
$\mathbf{2}$ & Milieu Therapy & 3.677 & Frequently \\
$\mathbf{3}$ & Self-Care Activities & 3.679 & Frequently \\
$\mathbf{4}$ & Psychobiologic Interventions And Health Teachings & 3.753 & Frequently \\
$\mathbf{5}$ & Case Management & 3.639 & Frequently \\
$\mathbf{6}$ & Health Promotion And Maintenance & 3.678 & Frequently \\
& Average & 3.684 & Frequently \\
\hline
\end{tabular}

Scaling: $\quad$ 4.21-5.00-"Whole", 3.41-4.20-"Frequent", 2.61-3.40-"Occasional" 1.81-2.60-"Rare", 1.00-1.80-"Never"

In terms of preliminary clinical care, the respondents perceive their extent of interventions in terms of the six (6) key areas of psychiatric care to be "frequently" emphasized. They described the extent of demonstration and performance of counseling, milieu therapy, self-care activities, psychobiologic interventions \& health-teachings, case management, health promotion and maintenance as generally emphasized in about $75 \%$ of the time in their respective clinical areas.

Table 3. Relationship Between Personal Profile and Extent of Clinical Care to Clients in terms of Health Promotion and Maintenance

\begin{tabular}{ccccc}
\hline \multicolumn{1}{c}{ Relationship } & $\begin{array}{c}\text { Correlation } \\
\text { coefficient }\end{array}$ & $\boldsymbol{p}$-value & Remarks \\
\hline Age & $\chi^{2}=5.607$ & 0.231 & Not significant \\
Gender & $\chi^{2}=1.219$ & 0.544 & Not significant \\
Civil Status & Health Promotion & $\chi^{2}=0.717$ & 0.699 & Not significant \\
Ethnic Affiliation & and Maintenance & $\chi^{2}=11.660$ & $0.020^{*}$ & Significant \\
Religion & & $\chi^{2}=3.168$ & 0.205 & Not significant \\
Monthly Salary & & $\chi^{2}=2.241$ & 0.691 & Not significant \\
Length of Service & $\chi^{2}=4.774$ & 0.311 & Not significant \\
\hline
\end{tabular}

* Significant at 0.05 level of significance

It can be gleaned from the above results that at 0.05 level of significance, ethnic affiliation was significantly related to the psychiatric care key areas of health promotion and maintenance implying that the respondents' ethnic and cultural 
background has an influence to the extent and degree of clinical nursing care rendered

\section{DISCUSSION}

Table 1 shows the sociodemographic profile of the eligible samples. There were more female nurses (88) in the study, taking about $77.90 \%$ of the total sample size. This finding is to be expected especially in the discipline of nursing practice. It is widely accepted that the profession is predominantly filled-up by a majority of female personnel. It is mostly ascribed to the phenomena of caring, which is generally attributed to be a motherly, feministic virtue. Even in the earlier times, nursing had been represented mostly by female figures such as the likes of Florence Nightingale. Hence, this finding largely coincides with the literature sources indicating that the nursing workforce, by and large, is mostly female (World Health Organization, 2003).

Regardless of gender, it is seen in several reports that there is an apparent insufficiency in the quantity of nurses who have expertise in psychiatric care.

In lieu of this, a survey conducted by the WHO in 2007 cited that most healthcare systems all over the world are struggling with the sequelae of having a very limited number of nursing manpower. There seems to be a continued challenge of recruiting more nurses into the practice at the same time making sure that those who are already employed remain to be absorbed in their field of work. It was also reported that the inability to hire nurses to serve in the mental health settings stems from a lack of interest in this specialty area and the inadequacy of available incentives grants for professionals working in the field. Furthermore, compounding the problem of nursing shortage in third-world countries is the emigration of nurses to developed countries to seek for better employment opportunities. It cripples the workforce of healthcare systems as the ones with more advanced experience in handling to clients with psycho-behavioral manifestations

psychiatric care are also the same ones who would be readily employed abroad. This exodus leaves behind a pool of novice practitioners in the home country. Other than emigration, there are also nurses who simply choose to leave the profession behind to pursue other fields of practice. Moreover, the lack of safety and security in the workplace and the stigma attached to the dynamics of mental disorders has unnecessarily complicated the mental health nursing shortage (Saxena et al., 2007).

Confounded by the interplay of several factors, the Philippines also manifest such a decline in the number of nursing practitioners who are employed as mental health personnel. Ocular surveys and regional reports show that there are very few nurses who have specialization in psychiatric nursing provided that there are also no mental health facilities in the province.

Majority of the respondents have an age bracket of 23 to 28 years and were mostly single $(71.70 \%)$. This indicates that most nurses in the study fall under the starting or entry-level nurses category. They are usually new to the practice as professional beginners after completing the four-year nursing degree. Hence, these nurses may have a limited experience in handling patients with psycho-behavioral symptoms. Particularly in the case of mental health care delivery, most finishers of the undergraduate degree in Nursing are considered generalist practitioners who are trained only with the basic and fundamental aspects of care for patients with psychiatric conditions.

This is particularly true at this point in time following the advent of patient deinstitutionalization (shifting of client care from hospital to community-based settings) where there is a growing primacy on making sure that mental health services become embedded in the mainstream medical services. This is increasingly 
becoming recognized because, typically, the point of entry for patients with complaints and manifestations of mental problem is the emergency rooms of most general hospitals. Therefore, it is undoubtedly important that nurses assigned in the emergency departments must also have specialist skills in the aspects of client assessment, management, and disposition (Wand, 2004).

Hence, further advanced trainings and specializations in mental health care services are needed in order for nurses who are in general acute care wards to provide streamlined interventions for the special needs of these unique types of clienteles.

Training and internship in mental health is not just an imperative prerequisite for the delivery of mental health care, it is also an essential requirement in the discharge of a total, holistic, and comprehensive general nursing care. Therefore, the need to further intensify the general nursing curricula should be put in place so that there will be a more reinforced incorporation of appropriate mental health components in the degree program (WHO, 2007).

Another study also showed that overall; both the undergraduate and postgraduate nursing curriculum should be enhanced vis-à-vis inclusion of mental health education contents. It is recommended that the number of hours apportioned to mental health must be raised and that psychiatry must be established as a priority course in the nursing program. Further recommendations included the following: the standardization of all training curriculum and exposure on a global level, inclusion of transdisciplinary influences in the current program, continuing education and other advanced degree courses in mental health, training more nurse educators to handle subjects and preceptorship programs, and the provision of more on-the-job training on topics relative to mental health promotion. Informants of the study also cited the gap between nurses with specializations and those without specializations in the psychiatric setting. Hence, mobility of students between countries through exchange study programs can be useful to help enhance learning opportunities for nursing students (Barrett et al., 2009).

Furthermore, the biggest portion of the respondents $(58.41 \%)$ in the study has a monthly income of P9, 000.00 and below per month. Majority $(72.60 \%)$ of them has Islam as their religion whilst the remaining fell in the Christianity and others category. In terms of ethnic affiliation, more than half $(65.50 \%)$ were Meranaos, and the rest of the samples represented the Cebuano, Maguindanaon and other tribes. This finding is to be expected considering that the research locale chosen is Lanao del Sur. The Province of Lanao del Sur, is a province in the Philippines located in the Bangsamoro Autonomous Region in Muslim Mindanao (BARMM). The people therefore are predominantly practitioners of Islam.

On the average, these nurses have served their current base hospitals for a length of 1 year and below (41.60\%). Consistent with the findings on the respondents present length of service, it is presumed that the reason why there is a high percentage of respondents who were employed under the non-regular status employment is that, most beginning nurses are not hired instantly for regular appointments in most hospital settings since they first go through a period of orientation and training to familiarize and slowly integrate into the roles and functions of a regular staff nurse.

Similarly, according to the WHO in 2003, physicians and nurses have very limited ability to appropriately recognize and manage mental disorders. It is seen that close to half $(41 \%)$ of all countries do not offer mental health training to their primary healthcare personnel.

Community-based providers are confronted with an array of factors and barriers in the delivery of mental healthcare. Two of the most common 
barriers identified are the insufficiency of available resources and the stigma associated with mental health. In general, understaffing is a recurrent issue in general health care, however, this is seen even more as a concern in the mental health care sector. Nurses usually receive minimum remuneration for the work that they do, however, even nurses with specialized experience and training in psychiatry are rarely compensated with higher salaries considering their advanced level of expertise. Even then, issues such as heavy workloads, high patient census, and low sense of physical safety and security all result to decreased level of quality in the care provided to clients. Hence, higher pays and more incentives for nurses specializing in mental health facilities are some of the proposed recommendations to elicit the delivery of better mental health care outcomes (Barrett et al., 2009).

Subsequently, through mentoring activities, most novice nurses gain the necessary competence and development that will help them prepare to step up to higher positions in the professional ladder (Gallego, 2018).

This is supported further in other literatures stating that "individuals who have been mentored report a variety of beneficial outcomes including higher promotion rates, salary, job and career satisfaction, organizational socialization, and organizational commitment, and lower job stress. In addition, the structure of the mentoring relationship appears to influence outcomes, with informal relationships generally being associated with more positive outcomes. Studies show that nurses who were recipients of mentoring activities reported having felt better career and psychosocial benefits comparable to those in the business and organizational behavior literatures" (Blastorah, 2009).

In summary, Table 2 illustrates that the nurse-respondents of the selected hospitals in Lanao del Sur generally considers the extent of implementation of the six nursing care areas tested under the dependent variable as frequently done. They described the extent of demonstration and performance of counseling, milieu therapy, self-care activities, psychobiologic interventions \& health-teachings, case management, health promotion and maintenance as generally emphasized in about $75 \%$ of the time in their respective clinical areas.

Aside from the above clinical care, Mental Health Care should contain 10 basic principles which are: Promotive and preventive activities for mental health; basic mental health accessibility; Mental health assessment (diagnosis, choice of treatment, determination of competence); mental healthcare institutions that provide the least restrictive environment; respect for the clients' right to self-determination; right to be provided with assistance; review procedures that are available for review and evaluation; Automatic periodic review; provision of qualified and informed decision maker; and right of the rule of law (WHO, 2000).

The present nursing service in acute care hospital provides training and exposure that are directed at improving the generalist competencies of nurses as clinicians in medical settings. Hence, in terms of psychiatric and mental health competency, most nurses are skeptical in their patterns of interaction with clients who have mental issues. They also express lack of confidence in terms of the skills needed to correctly assess the condition of patients. They also have limited understanding of the available services that can be given to clients with mental health issues (Wand \& Happell, 2001).

Since nurses perform a crucial role in the care of clients who are affected by mental illness; this role need to be highlighted and taken into account in the comprehensive mental health plans across all countries. By then, nurses who are equipped with the right practical and technical know-how in mental health care can already take on a wider array of 
responsibilities and capacities within the mental health services than is presently expected. Hence, their roles have to be expanded so that they can be able to demonstrate competencies in assessment, actual clinical care and follow-up utilizing their knowledge on appropriate psychopharmacologic interventions. Nurses must also have active participation and engagement in the policy-making and legislation process relative to the improvement of mental health care delivery. These roles are ultimately needed in countries where there are existing shortage of mental health care providers (Saxena et al., 2007).

Another important section is the determination of correlation between the sets of variables previously identified. Hence, attention is further focused on an attempt at identifying the relationship between the personal profiles of the respondents versus the extent of the preliminary clinical care that they provide to their psychologically vulnerable clients. The results are depicted in Table 3. As revealed in the findings, all personal profile variables, namely age, sex, civil status, religion, monthly income, type and size of family have no significant correlation with the extent of preliminary clinical care in terms counseling, milieu therapy, self-care activities, psychobiologic interventions, health-teachings, health promotion and maintenance. When tested at 0.05 level of significance, the $\mathrm{p}$-values obtained were all less than the critical value. Only the ethnic affiliation was significantly related to health promotion and maintenance.

This implies that the respondents' ethnic and cultural background has an influence to the extent and perhaps degree of clinical care that they can render to their clients with psycho-behavioral manifestations. Hence, the researcher is convinced that the implication of this findings be considered by institutions to particularly intensify and emphasize the need to encourage their nurses to become transculturally competent and sensitive to the diverse and unique cultural backgrounds of their clients with psychological disabilities.

According to a reviewed literature, the Filipino perception of mental illness is very much influenced by their inherited system of cultural beliefs and practices. Castro et al (2014) cited that other than the poor health service system in the local communities, the Filipinos' view on mental health also affects their reception and subscription to the programs provided thereof. Families of patients with mental illness would initially take it against the patient for suffering the condition. The patient is often regarded as a "weakling". Oftentimes the patient is thought to be just exaggerating the symptoms; invalidating them as simply "just in the mind". Given such impressions, the patient is expected to be just the one to provide remedy and take control over his feelings and thoughts. Furthermore, the stigma associated with having a mental illness is still very much embedded in the Filipino culture. Families tend to deny the existence of the affliction and would rather keep the condition to themselves thereby foregoing proper medical help.

People with mental disorders rely on their family members for care and support. Oftentimes, the family not only shoulders the expenses for the treatment, but they also offer physical and moral support. Statistics show that one in every four families has one member suffering from a form of mental illness. On top of the constraints put forth by the complications and distress of having to see a beloved family member being functionally crippled by a mental problem, family members also have to deal with the social stigma and discrimination in society. Social isolation and deprivation of the opportunity to participate in basic social networks in the community often result from being rejected by friends, neighbors and other important social figures (WHO, 2003).

Subsequently, it is also a common local belief that mental illness in the rural 
towns and regions are caused by spiritual powers and paranormal works. Traditional conceptions on curses and witchcrafts among community dwellers promote the subscription to indigenous faith healing practices rather than having the problem consulted to a health professional. For instance, a study about intellectual disability and treatment choices in the province of Negros Occidental revealed that among the local people, the preferred management of choice for mental illness was seeking the counsel of the suranho (traditional faith healer or shaman). Participants of the study stated that, habitually, family members would prefer referring the case of their loved one to a suranho under the belief that he can cast out the "evil spirit" that has taken over the body of their loved one who have been possessed by a ghost or a paranormal entity (Brolan $e t$ al., 2014).

The researcher also ascribe this significant correlation to the fact that most of the patients involved in the care are actually coming from the locale itself, communities of Lanao del Sur. As such, they are constituents of the Meranao community whose culture and practices are very unique and distinct from that of other tribes. Hence, in order for healthcare providers to be able to give the best, suitable, and apt psychobiologic interventions and health teachings to these patients, they have to also be exposed to trainings and conferences that deals much with the Meranao way of life, their perception of mental illness and their own approaches to mental health care.

Relatively, one respondent keenly depicted her experience in dealing with patients and expressed that: "I am Visayan (another Philippine tribe) and I am working here in Marawi City. What I find very challenging in my job is my inadequate exposure to the culture that they have here. Syempre dili ko taga diri, so kailangan ko makahibalo sa ilang culture, sa dialect ug uban pa. (Of course I am not from here, so I really need to learn about their culture, dialect and other things.) I have to be effective in my work, especially in handling psych patients. Lisod na! (It's hard). I think I need more education about them." (Nurse B from Hospital 4, Journal Response, August 18, 2016)

The giving of permanent mental health care services for patients with longstanding psychiatric illness has been, and continually is, one of the major issues confronting the delivery of mental health reforms in the past few years. This is commonly predicated to the following factors: most countries have no structured community services for mental health. In order to be able to formulate one, they have to contend with several barriers existing at the policy level sprouting from the lack of sufficient budget, not enough mental health policies and legislations, and the continued presence of procedural discrimination against persons with mental illness such as lack of insurance.

Other barriers have also been pointed out at the level of the health system, they include: difficulties in the issuance of financial resources from sponsoring agencies leading to under-investment in services at the community level; diminished extent of integration of mental services with the general health system; poor mergence of mental health and social care systems such as in the aspect of housing, social welfare, and employment opportunities; little coordinated partnership working between statutory and nonstatutory mental health services; and finally, the problem on the deficient training opportunities for health care practitioners across the systems (WHO, 2001).

\section{CONCLUSION}

The findings of this study suggest that the Philippines still has to continue on its effort to continuously improve its healthcare sector, paying particular emphasis on the aspect of mental health. The country has to tirelessly pursue and 
affirm the WHO vision of ensuring health for all, by making sure that health is inclusive in that, to speak of health is to speak of its encompassing coverage of not just physical but emotional, social, and psychological wellness converging in one body and system.

In order to make headway in terms of health, relevant legislations have to be enacted so that resources, processes and structures are set in place and health reforms be started in earnest as it is most necessary now more than ever. For it to be sustained, it is important to decentralize these reforms and make it visible across the entire country.

It is also seen that most developing countries worldwide need to have nurses who are adequately prepared and trained in the field of mental health care. Incidentally, the number of nurses without formal training in psychiatric practice is overwhelming high compared to those who have the experience. The role of nurses becomes more significant and crucial in view of this massive shortage in the professional workforce (WHO, 2007).

In the Lanao Sur province for instance, the research findings revealed that there is an insufficiency in the existing mental health training that are based on the understanding and adaptation of regional cultures for application in nursing care. The phenomenon of transcultural nursing is not only applicable to the ward settings in acute care for patients with physiologic complaints. The applicability of which is also visible in the care for clients with psychiatric needs.

Corollary to this, a growing body of trans-cultural evidence suggests that various psychological, social and behavioral factors can help facilitate the protection of health and support positive mental health. This protection is believed to support resistance and resilience against the acquisition of illnesses. It is also shown to diminish and delay the emergence of disabilities while helping enhance faster recovery time and recuperation from diseases. Some of these behavioral factors are: having secure attachment, practicing positive outlook in life, having adaptive coping strategies, achieving a sense of purpose and direction, being in control over one's decision and choices, having a satisfying and meaningful social relationships, and showing positive emotions (WHO, 2003).

Therefore, all nurses, even if they are assigned in non-psychiatric units, are still to be equipped with competencies in the care of patients who have symptomatologies that are associated with psycho-behavioral illness. All the more, nurses must also conduct a thorough assessment of the client's ethnic and cultural background and preferences so that specific and sensitive nursing actions are observed in provision of care. This can be ensured by training staff members to have the culturally-appropriate skills to work professionally and effectively with people who are vulnerable and at high risk of developing mental-behavioral disorders.

\section{REFERENCES}

Barrett, T. et al. (2009) 'Nurses are the key to improving mental health services in low- and middle-income countries: Short Communication', International Nursing Review. doi: $10.1111 / \mathrm{j} .1466-$ 7657.2008.00687.x.

Blastorah, M. (2009) 'The effect of mentoring on leadership selfefficacy in nurses', Lawrence $S$. Bloomberg Faculty of Nursing.

Brolan, C. E. et al. (2014) 'Suranho healing: Filipino concepts of intellectual disability and treatment choices in Negros Occidental', Disability and Society. doi: 10.1080/09687599.2013.771899.

Castro, N., Capinpin, L., Esteban A., Fojas, E., Luis, A. (2014). Issues on mental health legislation in the Philippines 
Conde, B. (2004) 'Philippines mental health country profile', International Review of Psychiatry. doi: 10.1080/095402603100016.

Fraenkel, J. R., Wallen, N. E. and Hyun, H. H. (2012) How to Design and Evaluate Research in Education, 8th Edition (2012), Climate Change 2013 - The Physical Science Basis.

Gallego, R. I. F. (2018) 'Structured Mentoring Strategies: Its Correlation to the Clinical, Leadership and Research Skills of Novice Nurses', JPAIR Multidisciplinary Research. doi: 10.7719/jpair.v33i1.605.

Hamid, H. et al. (2008) 'Evaluating the WHO Assessment Instrument for Mental Health Systems by comparing mental health policies in four countries', Bulletin of the World Health Organization. doi: 10.2471/BLT.07.042788.

Kane, C. F. (2015) 'The 2014 scope and standards of practice for psychiatric mental health nursing: Key updates', Online Journal of Issues in Nursing. doi: 10.3912/OJIN. Vol20No01Man01.

Patel, V. et al. (2016) 'Global Priorities for Addressing the Burden of Mental, Neurological, and Substance Use Disorders', in Disease Control Priorities, Third Edition (Volume 4): Mental, Neurological, and Substance Use Disorders. doi: 10.1596/978-1-4648-0426-7_ch1.

Saxena, S. et al. (2007) 'WHO'S assessment instrument for mental health systems: Collecting essential information for policy and service delivery', Psychiatric Services. doi: 10.1176/ps.2007.58.6.816.

'The world health report 2001 - Mental health: new understanding, new hope.' (2001) Bulletin of the World Health Organization. doi: 10.1590/S004296862001001100014.

Wand, T. (2004) 'Mental health liaison nursing in the emergency department: On-site expertise and enhanced coordination of care', Australian Journal of Advanced Nursing.

Wand, T. and Happell, B. (2001) 'The mental health nurse: Contributing to improved outcomes for patients in the emergency department', Accident and Emergency Nursing. doi: 10.1054/aaen.2000.0248.

World Health Organization, \& International Council of Nurses. (2007). Atlas: Nurses in mental health 2007

World Health Organization (2000) 'Mental health and work: Impact, issues and good practices', $\mathrm{WHO}$.

World Health Organization (2003) 'Guidelines nursing and midwifery workforce management', SEARO Technical Publication. 